\title{
Small-amplitude normal modes of a vortex in a trapped Bose-Einstein condensate
}

\author{
Marion Linn ${ }^{1,2}$ and Alexander L. Fetter ${ }^{1}$ \\ ${ }^{1}$ Department of Physics, Stanford University, Stanford, CA 94305-4060 \\ ${ }^{2}$ Physikalisches Institut, Universität Bonn, Nußallee 12, D-53115 Bonn, Germany
}

(March 10, 2021)

\begin{abstract}
We consider a cylindrically symmetric trap containing a small Bose-Einstein condensate with a singly quantized vortex on the axis of symmetry. A time-dependent variational Lagrangian analysis yields the small-amplitude dynamics of the vortex and the condensate, directly determining the equations of motion of the coupled normal modes. As found previously from the Bogoliubov equations, there are two rigid dipole modes and one anomalous mode with a negative frequency when seen in the laboratory frame.
\end{abstract}

PACS number(s): 03.75.F, 05.30.Jp, 32.80.Pj, 67.40.Db

\section{INTRODUCTION}

After the achievement of Bose-Einstein condensates in trapped atomic gases [1 3, interest in these systems has increased dramatically. An especially intriguing question concerns condensates containing a vortex. The lowenergy normal modes are important in characterizing the behavior of such a system. They have been studied extensively [5 11, mostly with the Bogoliubov equations [12] that involve quantum-mechanical normal-mode amplitudes. Consequently, the associated dynamical motion of the vortex core and the condensate center of mass can only be obtained indirectly. Here, we use a variational Lagrangian procedure to provide a more direct and intuitive treatment of the small-amplitude normal modes of a small (weakly interacting) Bose-Einstein condensate containing a singly quantized vortex. As in previous applications [8, 13], this approach yields a clear physical picture of the dynamics. We show that it reproduces the three lowest modes as found from the more intricate Bogoliubov analysis, thereby clarifying the physical interpretation. This description is particularly interesting for the anomalous mode that causes the local instability of the vortex in a trap at rest [5,6, 10].

The article is organized as follows: in Sec. II we derive the effective Lagrangian for a slightly off-center vortex in a small condensate. In the next section we solve the corresponding equations of motion for the vortex and the condensate and discuss the physical interpretation. Finally we summarize our results in the conclusion.

\section{EFFECTIVE LAGRANGIAN}

We consider a small Bose-Einstein condensate with $N$ particles containing a singly quantized vortex on the axis of symmetry, and observed in a frame rotating with an angular velocity $\Omega$. The harmonic trap is axisymmetric, with radial and axial frequencies $\omega_{\perp}$ and $\omega_{z}$ (the ratio $\lambda=\omega_{z} / \omega_{\perp}$ characterizes the axial asymmetry). The $s$ wave scattering length $a$ (assumed positive) characterizes the interparticle interactions. For a general condensate wave function $\Psi$, the Lagrangian for such a system is given by

$$
\begin{aligned}
L(\Psi)=\int d V & {\left[\frac{i}{2}\left(\Psi^{*} \partial_{t} \Psi-\Psi \partial_{t} \Psi^{*}\right)\right.} \\
& \left.-\Psi^{*}\left(H_{0}-\Omega L_{z}\right) \Psi-2 \pi \gamma|\Psi|^{4}\right],
\end{aligned}
$$

where $H_{0}$ is the Hamiltonian for the noninteracting condensate

$$
H_{0}=\frac{1}{2}\left[-\partial_{x}^{2}-\partial_{y}^{2}+\left(x^{2}+y^{2}\right)+\lambda\left(-\partial_{z}^{2}+z^{2}\right)\right],
$$

and $L_{z}=-i\left(x \partial_{y}-y \partial_{x}\right)$ is the $z$-component of the angular-momentum operator. All quantities are expressed in dimensionless units (the radial and axial coordinates are scaled with the radial and axial oscillator lengths $d_{\perp}=\sqrt{\hbar / M \omega_{\perp}}$ and $d_{z}=\sqrt{\hbar / M \omega_{z}}$, and frequencies are scaled with the radial trap frequency $\omega_{\perp}$ ). The condensate wave function $\Psi$ is normalized to unity and $\gamma \equiv N a / d_{z}$ is the small interaction parameter.

Next we construct a trial condensate wave function. The condensate wave function is assumed to be unchanged with respect to the noninteracting case along the axis of symmetry, which allows us to use the groundstate gaussian $\varphi_{0}(z)=\pi^{-1 / 4} \exp \left(-z^{2} / 2\right)$. The radial wave function is based on the vortex ground state, namely a two-dimensional gaussian times the vortex factor $x+i y=r e^{i \varphi}$. We are interested in the relative motion of the vortex which requires introducing time-dependent parameters for the vortex position. In the weak-coupling limit, the radius of the vortex core is comparable to the radius of the condensate, so that the displacement $\mathbf{r}_{0}(t)=\left[x_{0}(t), y_{0}(t)\right]$ of the vortex, the displacement $\mathbf{r}_{1}(t)=\left[x_{1}(t), y_{1}(t)\right]$ of the condensate, and the induced velocity of the condensate $\boldsymbol{\alpha}(t)=\left[\alpha_{x}(t), \alpha_{y}(t)\right]$ must all be included. Hence we use the following trial function

$$
\begin{aligned}
\Psi_{v}(x, y, z, t) & =\frac{C}{\pi^{3 / 4}}\left[\left(x-x_{0}\right)+i\left(y-y_{0}\right)\right] \\
& \times e^{-\frac{1}{2}\left[\left(x-x_{1}\right)^{2}+\left(y-y_{1}\right)^{2}+z^{2}\right]} e^{i\left(\alpha_{x} x+\alpha_{y} y\right)},
\end{aligned}
$$


where $C^{-2}=1+\left|\mathbf{r}_{1}-\mathbf{r}_{0}\right|^{2}$. Since we focus on the dynamical motion of the vortex and the condensate, we ignore the possibility of monopole or quadrupole modes, and the dimensionless gaussian widths are taken as one. The same trial function, Eq. (3), also serves to characterize the stability of the vortex in a small condensate [14].

In evaluating the integration in $L\left(\Psi_{v}\right)$, we retain all terms up to second order in the time-dependent parameters, yielding

$$
\begin{aligned}
L_{\text {eff }} & =-2-\frac{\lambda}{2}-\frac{\gamma}{2 \sqrt{2 \pi}}+\Omega \\
& -\dot{\alpha}_{x}\left(2 x_{1}-x_{0}\right)-\dot{\alpha}_{y}\left(2 y_{1}-y_{0}\right) \\
& -\dot{x}_{0}\left(y_{1}-y_{0}\right)+\dot{y}_{0}\left(x_{1}-x_{0}\right) \\
& -\frac{1}{2}\left(x_{1}^{2}+y_{1}^{2}\right)-x_{0}\left(x_{1}-x_{0}\right)-y_{0}\left(y_{1}-y_{0}\right) \\
& -\frac{1}{2}\left(\alpha_{x}^{2}+\alpha_{y}^{2}\right)+\alpha_{x}\left(y_{1}-y_{0}\right)-\alpha_{y}\left(x_{1}-x_{0}\right) \\
& +\Omega\left[x_{0}\left(x_{1}-x_{0}\right)+y_{0}\left(y_{1}-y_{0}\right)\right. \\
& \left.-\alpha_{x}\left(2 y_{1}-y_{0}\right)+\alpha_{y}\left(2 x_{1}-x_{0}\right)\right] \\
& -\frac{\gamma}{\sqrt{2 \pi}}\left[\left(x_{1}-x_{0}\right)^{2}+\left(y_{1}-y_{0}\right)^{2}\right]
\end{aligned}
$$

This Lagrangian takes a simpler form when written in terms of new variables $\boldsymbol{\delta}=\boldsymbol{r}_{1}-\boldsymbol{r}_{0}$ and $\boldsymbol{\epsilon}=2 \boldsymbol{r}_{1}-\boldsymbol{r}_{0}$ :

$$
\begin{aligned}
L_{\mathrm{eff}} & =-2-\frac{\lambda}{2}-\frac{\gamma}{2 \sqrt{2 \pi}}+\Omega \\
& -\dot{\alpha}_{x} \epsilon_{x}-\dot{\alpha}_{y} \epsilon_{y}-\left(\dot{\epsilon}_{x}-2 \dot{\delta}_{x}\right) \delta_{y}+\left(\dot{\epsilon}_{y}-2 \dot{\delta}_{y}\right) \delta_{x} \\
& -\frac{1}{2} \alpha^{2}+\alpha_{x}\left(\delta_{y}-\Omega \epsilon_{y}\right)+\alpha_{y}\left(-\delta_{x}+\Omega \epsilon_{x}\right) \\
& -\frac{1}{2} \epsilon^{2}+\Omega \boldsymbol{\delta} \cdot \boldsymbol{\epsilon}+\left(-2 \Omega+\frac{3}{2}-\frac{\gamma}{\sqrt{2 \pi}}\right) \delta^{2} .
\end{aligned}
$$

\section{NORMAL MODES}

The effective Lagrangian leads to six coupled differential equations for the displacements and the induced velocity. Elimination of the latter through

$$
\boldsymbol{\alpha}=\dot{\boldsymbol{\epsilon}}+\hat{\boldsymbol{z}} \times(\Omega \boldsymbol{\epsilon}-\boldsymbol{\delta})
$$

yields two uncoupled pairs of homogeneous equations for the displacements $\boldsymbol{\delta}$ and $\boldsymbol{\epsilon}$ :

$$
\begin{aligned}
\left(1-\frac{\gamma}{2 \sqrt{2 \pi}}-\Omega\right) \delta_{y}(t)+\dot{\delta}_{x}(t) & =0, \\
-\left(1-\frac{\gamma}{2 \sqrt{2 \pi}}-\Omega\right) \delta_{x}(t)+\dot{\delta}_{y}(t) & =0, \\
\left(\Omega^{2}-1\right) \epsilon_{x}(t)+2 \Omega \dot{\epsilon}_{y}(t)-\ddot{\epsilon}_{x}(t) & =0, \\
\left(\Omega^{2}-1\right) \epsilon_{y}(t)-2 \Omega \dot{\epsilon}_{x}(t)-\ddot{\epsilon}_{y}(t) & =0 .
\end{aligned}
$$

The $\boldsymbol{\delta}$ mode with $\boldsymbol{\epsilon}=0$ will be seen to correspond to the anomalous mode; it has $\boldsymbol{r}_{0}=2 \boldsymbol{r}_{1}$ (so that the displacement of vortex is twice that of the condensate). In contrast, the $\boldsymbol{\epsilon}$ mode with $\boldsymbol{\delta}=0$ represents a rigid dipole oscillation with $\boldsymbol{r}_{0}=\boldsymbol{r}_{1}$ (so that the vortex and the condensate move together).

The physically relevant solutions of the equation of motion must represent quantum states with positive normalization. A convenient condition to check this normalization is [7]

$$
\int d V i\left(n^{\prime *} \Phi^{\prime}-\Phi^{\prime *} n^{\prime}\right)=\frac{\hbar}{M},
$$

where $n^{\prime}$ and $\Phi^{\prime}$ are the complex fluctuations of the particle density and the velocity potential respectively. The density fluctuations are obtained by expanding the density $n=\left|\Psi_{v}\right|^{2}=n_{0}+\delta n$, where $n_{0}=$ $\left(r^{2} / \pi^{3 / 2}\right) \exp \left(-r^{2}\right) \exp \left(-z^{2}\right)$ is the unperturbed density for the condensate with a vortex. We find

$$
\delta n \approx 2 n_{0} \boldsymbol{r} \cdot\left(\boldsymbol{r}_{1}-\frac{\boldsymbol{r}_{0}}{r^{2}}\right),
$$

and use of the normal-mode amplitudes yields the expression in plane polar coordinates

$$
\begin{aligned}
\delta n=2 n_{0} & \left\{\cos \varphi\left[r\left(\epsilon_{x}-\delta_{x}\right)-r^{-1}\left(\epsilon_{x}-2 \delta_{x}\right)\right]\right. \\
& \left.+\sin \varphi\left[r\left(\epsilon_{y}-\delta_{y}\right)-r^{-1}\left(\epsilon_{y}-2 \delta_{y}\right)\right]\right\} .
\end{aligned}
$$

Given the explicit form of the displacements $\boldsymbol{\delta}$ and $\boldsymbol{\epsilon}$ (see below), this real expression can be converted to complex density fluctuations through

$$
\delta n=n^{\prime} e^{-i \omega t}+n^{\prime *} e^{i \omega t} .
$$

The complex fluctuations in the velocity potential follow by expanding the phase of the condensate wave function $S=\arctan \left[\left(y-y_{0}\right) /\left(x-x_{0}\right)\right]+\boldsymbol{\alpha} \cdot \boldsymbol{r} \approx \varphi+\delta S$, since

$$
\delta \Phi=(\hbar / M) \delta S=\Phi^{\prime} e^{-i \omega t}+\Phi^{\prime *} e^{i \omega t} .
$$

The vortex part $\delta S_{v}$ of this phase perturbation is $\delta S_{v}=$ $\left(-y_{0} x+x_{0} y\right) / r^{2}$, and condensate-velocity part $\delta S_{\alpha}=\boldsymbol{\alpha} \cdot \boldsymbol{r}$ can be rewritten with Eqs. (6). In terms of the normal mode variables $\boldsymbol{\delta}$ and $\boldsymbol{\epsilon}$, we find

$$
\begin{aligned}
\delta S & =\cos \varphi\left[r \dot{\epsilon}_{x}-\epsilon_{y}\left(\frac{1}{r}+r \Omega\right)+\delta_{y}\left(\frac{2}{r}+r\right)\right] \\
& +\sin \varphi\left[r \dot{\epsilon}_{y}+\epsilon_{x}\left(\frac{1}{r}+r \Omega\right)-\delta_{x}\left(\frac{2}{r}+r\right)\right],
\end{aligned}
$$

and this expression can readily be converted into the complex velocity potential $\Phi^{\prime}$.

The equations of motion for the anomalous mode $\boldsymbol{\delta}$ involve the characteristic frequency

$$
\omega_{a}=-1+\frac{\gamma}{2 \sqrt{2 \pi}}+\Omega,
$$

and the solution has the form

$$
\boldsymbol{\delta}(t)=\frac{1}{\sqrt{2}} \delta_{0}\left(\cos \omega_{a} t,-\sin \omega_{a} t\right)
$$


where $\delta_{0}$ is an infinitesimal amplitude and the factor $1 / \sqrt{2}$ fixes the normalization of the perturbation amplitudes (see below). Since $\epsilon=0$ for this mode, the real small perturbation in the density becomes

$$
\delta n_{a}=-2 n_{0}\left(r-\frac{2}{r}\right)\left(\cos \varphi \delta_{x}+\sin \varphi \delta_{y}\right),
$$

and the complex density fluctuation (with time dependence $\left.e^{-i \omega_{a} t}\right)$ is

$$
n_{a}^{\prime}=-\frac{\delta_{0}}{\sqrt{2}} n_{0}\left(r-\frac{2}{r}\right) e^{-i \varphi} .
$$

As expected from the physical picture of a single-particle transition from the vortex condensate with unit angular momentum per particle to the unoccupied axisymmetric gaussian ground state, the quantum number of the $z$ component of the angular momentum in this mode is $m=-1$. The real phase fluctuation for the anomalous mode is

$$
\delta S_{a}=\left(\frac{2}{r}+r\right)\left(\cos \varphi \delta_{y}-\sin \varphi \delta_{x}\right),
$$

with the corresponding complex velocity potential

$$
\Phi_{a}^{\prime}=\frac{\delta_{0} \hbar}{2 \sqrt{2} i M}\left(\frac{2}{r}+r\right) e^{-i \varphi} .
$$

For this anomalous mode, it is easy to verify that the normalization integral (11) has the proper positive value. Since $\epsilon=0$, Eq. (18) shows that the amplitudes of the vortex $\boldsymbol{r}_{0}$ and the condensate $\boldsymbol{r}_{1}$ become

$$
\begin{aligned}
& \boldsymbol{r}_{0}=\sqrt{2} \delta_{0}\left(-\cos \omega_{a} t, \sin \omega_{a} t\right), \\
& \boldsymbol{r}_{1}=\frac{1}{2} \sqrt{2} \delta_{0}\left(-\cos \omega_{a} t, \sin \omega_{a} t\right) ;
\end{aligned}
$$

they both execute small-amplitude in-phase motion with $\boldsymbol{r}_{0}$ twice as large as $\boldsymbol{r}_{1}$. For $\Omega=0$, the anomalous frequency (17) is negative, and the motion is counterclockwise in a positive direction (namely right-circular motion with positive helicity). With increasing rotation frequency $\Omega$, the negative anomalous frequency increases towards zero and vanishes at the metastable rotation frequency $\Omega_{m}=1-\gamma /(2 \sqrt{2 \pi})$ 14]. For $\Omega>\Omega_{m}$, the apparent motion becomes clockwise (namely left-circular motion in a negative direction). The expressions found here for the density and velocity potential fluctuations $n_{a}^{\prime}$ and $\Phi_{a}^{\prime}$ coincide with those found from the Bogoliubov approach [6]. If we had omitted either the induced velocity or the displacement of the condensate, we would have found different frequencies, just as in the discussion of vortex stability [14. The present Lagrangian treatment has the advantage of providing a physical picture of the motion, clarifying the nature of the anomalous mode. In the weak-coupling limit, the vortex and condensate move in phase, in contrast to the behavior found in the strongcoupling (Thomas-Fermi) limit [8].
In a similar manner, we can treat the dipole modes, which have $\boldsymbol{\delta}=0$, so that $\boldsymbol{r}_{0}=\boldsymbol{r}_{1}$ and the vortex moves rigidly with the condensate. Equations (9) and (10) show that the $x$ and $y$ motions are uncoupled if $\Omega=0$. In general, however, there are two coupled normal modes with frequencies $\omega_{+}=1-\Omega$ and $\omega_{-}=1+\Omega$. The notation $\omega_{ \pm}$ reflects the general construction of the Bogoliubov amplitudes $u_{ \pm}=a_{ \pm}^{\dagger} \Psi$ and $v_{ \pm}=a_{\mp} \Psi^{*}$ for the dipole modes in the laboratory frame, where $\Psi$ is any solution of the Gross-Pitaevskii equation in an axisymmetric trap; the resulting density perturbation is $n_{ \pm}^{\prime} \propto e^{ \pm i \varphi} e^{-i \omega_{ \pm} t}$, see Eqs. (28) and (30) below. It is easy to see that the general solution is a linear combination of the two distinct normal modes

$$
\begin{aligned}
& \boldsymbol{\epsilon}_{+}(t)=\epsilon_{+}(0)\left(\cos \omega_{+} t, \sin \omega_{+} t\right) \\
& \boldsymbol{\epsilon}_{-}(t)=\epsilon_{-}(0)\left(\cos \omega_{-} t,-\sin \omega_{-} t\right)
\end{aligned}
$$

where $\epsilon_{+}(0), \epsilon_{-}(0)$ are infinitesimal small amplitudes.

We can now check the normalization for the dipole modes. The first-order contribution to the density for both modes is given by

$$
\delta n_{ \pm}=2 n_{0}\left(r-\frac{1}{r}\right)\left(\cos \varphi \epsilon_{x \pm}+\sin \varphi \epsilon_{y \pm}\right),
$$

and hence the complex density fluctuations are

$$
n_{ \pm}^{\prime}=\epsilon_{ \pm}(0) n_{0}\left(r-\frac{1}{r}\right) e^{ \pm i \varphi} .
$$

For the phase fluctuations we find

$$
\delta S_{ \pm}=\left[\frac{1}{r}+r\left(\Omega \pm \omega_{ \pm}\right)\right]\left(\sin \varphi \epsilon_{x}-\cos \varphi \epsilon_{y}\right),
$$

and the resulting complex fluctuations for the velocity potential are

$$
\Phi_{ \pm}^{\prime}=\frac{\epsilon_{ \pm}(0) \hbar}{2 i M}\left(r \pm \frac{1}{r}\right) e^{ \pm i \varphi},
$$

independent of the external rotation frequency $\Omega$. With these expressions, the normalization integral is one for both normal modes with frequencies $\omega_{ \pm}$.

The final expression for the motion of the vortex and the condensate in the dipole modes $\left[\boldsymbol{r}(t) \equiv \boldsymbol{r}_{0}(t)=\boldsymbol{r}_{1}(t)\right]$ is

$$
\boldsymbol{r}_{ \pm}(t)=r_{0}\left(\cos \omega_{ \pm} t, \pm \sin \omega_{ \pm} t\right)
$$

with an infinitesimal amplitude $r_{0}$. We again find circular trajectories, and here they are the same for the vortex and the condensate motion, confirming that the modes are indeed the rigid dipole oscillations of the vortex and the condensate. For stability reasons, only the region $|\Omega|<1$ is relevant; thus the motion is always counter-clockwise (positive) for $\omega_{+}$and clockwise (negative) for $\omega_{-}$. Comparison with the Bogoliubov results [6] 
shows exact agreement for $\Omega=0$. The rotation splits the dipole frequencies by twice the external rotation frequency. This result can be visualized by expressing the dipole modes (for $\Omega=0$ ) in terms of circular quanta, namely as circular motions in the positive and negative sense. For zero external rotation, the angular velocities are degenerate. In a frame rotating with $\Omega$ in the positive sense, the positive mode rotates at the difference $1-\Omega$ and hence slows down. The negative mode, however, speeds up by the same amount, leading to an effective splitting in the rotating frame of $2 \Omega$.

\section{CONCLUSION}

We have shown that a time-dependent Lagrangian approach provides physical insight into the nature of the low-energy modes of a vortex in a small trapped BoseEinstein condensate. The anomalous mode is an in-phase circular motion of the vortex and the condensate with different amplitudes. In a rotating frame, the sense of this mode reverses from positive to negative when the external rotation frequency exceeds the metastable frequency [14]. The dipole modes are rigid rotations of the vortex and the condensate together. In terms of the density and phase fluctuations, all these results agree exactly with those from the Bogoliubov approach [6].

\section{ACKNOWLEDGMENTS}

This work was supported in part by NSF Grant No. 94-21888 and by the DAAD (German Academic Exchange Service) "Doktorandenstipendium im Rahmen des gemeinsamen Hochschulsonderprogramms III von Bund und Ländern" (M. L.). A. L. F. thanks the Aspen Center for Physics where part of this work was performed.

[1] M. H. Anderson, J. R. Ensher, M. R. Matthews, C. E. Wieman, and E. A. Cornell, Science 269, 198 (1995).

[2] K. B. Davis, M.-O. Mewes, M. R. Andrews, N. J. van Druten, D. S. Durfee, D. M. Kurn, and W. Ketterle, Phys. Rev. Lett. 75, 3969 (1995).

[3] C. C. Bradley, C. A. Sackett, and R. G. Hulet, Phys. Rev. Lett. 78, 985 (1997).

[4] G. Baym and C. J. Pethick, Phys. Rev. Lett. 76, 6 (1996).

[5] R. J. Dodd, K. Burnett, M. Edwards, and C. W. Clark, Phys. Rev. A 56, 587 (1997).

[6] A. L. Fetter, J. Low Temp. Phys. 113, 198 (1998).

[7] A. A. Svidzinsky and A. L. Fetter, Phys. Rev. A 58, 3168 (1998).
[8] A. A. Svidzinsky and A. L. Fetter, cond-mat/9811348.

[9] F. Zambelli and S. Stringari, Phys. Rev. Lett. 81, 1754 (1998).

[10] T. Isoshima and K. Machida, J. Phys. Soc. Jpn. 68, 487 (1999).

[11] J. J. García-Ripoll and V. M. Pérez-García, condmat/9903353.

[12] N. Bogoliubov, J. Phys. (Moscow) 11, 23 (1947).

[13] V. M. Pérez-García, H. Michinel, J. I. Cirac, M. Lewenstein and P. Zoller, Phys. Rev. A 56, 1424 (1997).

[14] M. Linn and A. L. Fetter, cond-mat/9906139. 